\title{
LA INNOVACION EN EL SERVICIO AL CLIENTE
}

\author{
ADRIANA MARCELA GONZALEZ PALACIOS \\ UNIVERSIDAD SANTO TOMAS
}

\section{RESUMEN}

La importancia que ha cobrado un buen servicio al cliente es fundamental en cualquier empresa, sea de servicios o de cualquier otro sector. El servicio al cliente es la pieza innovadora que todas las empresas a nivel mundial de hace unos 10 años para acá, han logrado identificar como factor esencial para el éxito de sus negocios. Pero que significa un buen servicio al cliente?, "Es el manejo de la satisfacción a través de producir percepciones positivas del servicio..." (Nudel, 2009); es decir que la opinión de los clientes es como una evaluación de la empresa y de los productos y servicios que se estén brindando.

Existen variables que terminan la importancia de prestar un excelente servicio al cliente de las cuales algunas son, la competencia es un factor directamente proporcional al grado de valor agregado ofrecido a los clientes finales, los clientes cada día son más exigentes tanto en los productos como en el servicio brindado por la compañía, y si un cliente queda satisfecho con el servicio recibido las probabilidades de que vuelva y haga buena propaganda de la compañía, es muy alta, (K., 2014). Estas variables anteriormente mencionadas indican que el servicio al cliente se ha convertido en un plus que cada día se incrementa en las empresas y está muy conectado al Know How de cada compañía.

En este trabajo se hará un análisis de comparación entre dos grandes instituciones, que ofrecen servicios a un nicho de mercado especifico, con muy poca competencia, lo cual hace que el tipo de servicio al cliente no sea el más óptimo ya que la exigencia de este nicho no es muy alta. La empresa que se analizó en la visita a España, es Barcelona Activa, la cual se ha caracterizado por brindar un excelente servicio al cliente a lo largo de su permanencia en el mercado local e internacional. Y por parte de Colombia se analizara a la Cámara de Comercio de Bogotá, la cual durante su larga trascendencia en el país ha hecho unos logros altamente importantes en el país y en el desarrollo de la ciudad de Bogotá. 


\section{TABLA DE CONTENIDOS}

1. INTRODUCCION

2. OBJETIVO 4

2.1 OBJETIVO GENERAL__ 4

2.2 OBJETIVOS ESPECIFICOS__ 4

3. MARCO CONCEPTUAL__ 4

3.1 LA CIENCIA DE LOS SERVICIOS: UN NUEVO ENFOQUE PARA LA

INNOVACION EN COMPANIAS DEL SERVICIO.__ 4

3.2 EN BUSCA DE UN SERVICIO MEJOR: LA CLAVE ES INNOVAR.___ 5

3.3 IMPLICACIONES DE LA ORIENTACION PROACTIVA HACIA EL MERCADO,

LA COOPERACION Y EL USO DE LAS TIC EN LOS PROCESOS DE

INNOVACION DE PRODUCTOS Y SEVICIOS.__ 6

3.4 ENFOQUE METODOLOGICO PARA LA INNOVACION EN EMPRESAS._ 6

3.5 EL PROCESO DE COCRECION DE VALOR Y SU IMPACTO EN LA ESTRATEGIA DE INNOVACION EN EMPRESAS DE SERVICIO.__ 7

4. METODOLOGIA___ 8

5. RESULTADOS__ 9

6. CONCLUSIONES__ 10

7. BIBLIOGRAFIA____ 11

ANEXOS

\section{INTRODUCCION}

El servicio al cliente es un elemento esencial en todas las empresas de cualquier sector, ya que es la percepción que tiene su stakeholders principal y por el cual la empresa funciona, se estanca o crece; y es por esto y por otras razón más como la competencia, la variedad de productos y servicios en el mercado y la agresividad del mercadeo que el servicio al cliente que se brinda debe ser el mejor para que así el cliente se convenza que la mejor opción del mercado es esa determinada compañía. 
Con este trabajo de grado se pretende analizar los distintos y nuevos mecanismos de innovación que algunas empresas españolas, como Barcelona Activa, implementan para el servicio con sus clientes finales.

Por medio de la visita a la empresa Barcelona Activa, que es una empresa enfocada principalmente en el servicio al cliente, se quiere identificar cuáles son los puntos más favorables y decisivos para impartir un excelente servicio al cliente y de esa misma manera poder recibir por parte del cliente la fidelización a sus servicios.

El servicio al cliente es sumamente importante hoy en día, ya que el medio de competitividad en el que las empresas se encuentran hoy por hoy, hace que este ítem (servicio), sea un plus que se le da al cliente pero finalmente es lo que decide si este sigue con esa determinada empresa, prestadora del servicio, o se vaya con la competencia, dado que los productos como tales ya no son tan exclusivos como antes.

La empresa Barcelona Activa nace en el año de 1986 en el Ayuntamiento de Cataluña, bajo los parámetros de las políticas de promoción económica de dicho ayuntamiento. Lleva más de 25 años ayudando a impulsar el nuevo surgimiento de empresas apoyando el nacimiento de innovadores proyectos económicamente prometedores y a la consecución de personas desempleadas. (Activa, 2014)

Llamo la atención esta empresa española, ya que su trascendencia a nivel nacional ha tenido un gran impacto no solo en Cataluña, sino en toda España, dado que los proyectos de emprendimiento que allí se crean y surgen, se han desarrollado en su mayoría en el sector de servicios, y son proyectos exitosos, debido a la oportunidad económica que están brindando en ese país es alta, e importante, teniendo en cuenta que España ha venido saliendo de una crisis económica que afecto directamente la tasa de desempleo, y los resultados optimistas que Barcelona Activa, con sus dos grandes iniciativas ha brindado a esa economía un gran soporte y apalancamiento, porque este sector terciario es uno de los que más mano de obra emplea (Antonio, 2011), en comparación al sector primario y secundario de la economía.

Esta gran empresa ha tenido varios reconocimientos tanto nacional como internacionalmente por su políticas innovadoras de apoyo a las empresas y al empleo, en grandes instituciones tales como la Comisión Europea, la OCDE, la Eurocities, la red Europea de Incubación Empresarial EBN; y también reconocimiento en ciudades Latinoamericanas como Monterey, Bogotá, Buenos Aires, Quito, entre otras. (Ana, 2010)

La finalidad de este documento es determinar por medio del análisis de la gestión que ha desempeñado Barcelona Activa, cuáles pueden ser los elementos fundamentales para la innovación en el servicio, ya que ha sido una empresa destacada por esto a nivel nacional e internacional, y así poder tomarlos como base para nuevos proyectos de emprendimiento en Colombia y en empresas de servicios, dado que es un sector muy importante en la economía en general. 


\section{OBJETIVOS}

\subsection{OBJETIVO GENERAL}

Determinar cuáles son los factores claves de éxito de la empresa Barcelona Activa, como empresa prestadora de servicios e impulsadora de nuevos empresarios y de empleos en el Ayuntamiento de Barcelona, para aplicarlos en empresas colombianas prestadoras de servicios.

\subsection{OBJETIVOS ESPECIFICOS}

$\checkmark$ Analizar como es el sistema de interacción con sus clientes finales para lograr su éxito en con las nuevas empresas.

$\checkmark$ Comparar como es sistema de servicio al cliente que emplean empresas colombianas similares a Barcelona Activa.

Examinar cuales pueden ser los factores idóneos para lograr un servicio al cliente de excelente calidad, acorde al ambiente y competitivo para la población colombiana.

\section{MARCO CONCEPTUAL}

En este marco conceptual basado en 5 articulo científicos y académicos, se pretende esbozar cual es la importancia del servicio al cliente, y así poder comprender lo fundamental que es la innovación en este factor, el cual se está convirtiendo en la columna vertebral de la mayoría de las empresas.

\subsection{LA CIENCIA DE LOS SERVICIOS: UN NUEVO ENFOQUE PARA LA INNOVACION EN COMPANIAS DEL SERVICIO.}

El conocido sector terciario, ha venido en un gran crecimiento en los últimos años en países como Estados Unidos, donde se ha visto un incremento por este sector de la economía, donde empresas como IBM (Martinez Angel, 2007) han incrementado su preocupación por esta parte de la economía. Es por ello que su preocupación ya tiene nombre, "Ciencia de los Servicios", y para ello ha creado nuevos lazos con prestigiosas universidades que también ya se están preocupando por el mismo tema, para trabajar en conjunto con los más destacados estudiantes, que cumplan con unas características específicas para que empiecen a trabajar en nuevos proyectos para la compañía, aun sin haberse graduado de la universidad. 
La ciencia de los Servicios más específicamente viene siendo la forma de crear un conocimiento que genera valor empresarial. Es por esto que hay varios actores interesados en que esta "Ciencia del Servicio" se formalice y se profundice, de tal manera, que los próximos profesionales en este ramo, sean profesionales íntegros, es decir tengan competencias tales como, tecnólogos (elementos de tecnología), negociadores (aspectos económicos, y de marketing), y socios-organizativos(aspectos multiculturales), (Martinez Angel, 2007). Al anterior tipo de perfil profesional, es al que se le puede denominar Agente innovador, ya que debe tener la capacidad de sintetizar muchos conocimientos, en los campos anteriormente nombrados, para poder potencializar la transformación en la sociedad.

Este concepto aquí presentado, ya desde hace unos años está siendo tratado por prestigiosas universidades tales como University of California Berkeley, la Arizona State University, Universiteit van Amsterdam, Univerisity of Dublin, University of Manchester, (Martinez Angel, 2007), donde desde el 2006 trabajando en conjunto por el tema de la Ciencia del Servicio.

\subsection{EN BUSCA DE UN SERVICIO MEJOR: LA CLAVE ES INNOVAR.}

En la última década el sector terciario, el de los servicios, ha tomado una gran importancia, ya que de gran parte del servicio que las compañías prestan a sus clientes depende que estos vuelvan a la empresa o no, convirtiéndose en un elemento fundamental para que los gerentes actuales tomen el servicio como un factor de éxito y competitividad en los negocios.

Por ejemplo, en el caso de las compañías que prestan servicios de asesorías, la estrategia que allí aplican es la diferenciación en la prestación del servicio y un constante cambio en la manera de presentar el producto o resultado de la consultoría, lo cual se traduce en una constante innovación del servicio.

La innovación en el servicio es relativamente un tema nuevo, tal y como lo menciona (Sundbo, 1997), existen tres grandes impulsores de la innovación para el servicio 1) el involucramiento de nuevas tecnologías. 2) nuevas decisiones y creatividad del recurso humano de la organización. 3) la relación entre las estrategias de la compañía y la innovación; y para que ellas puedan rendir frutos en dicha organización hay que ponerlas en marcha para el bienestar de los resultados del servicio. 
Es claro que para que las compañías tengan un verdadero éxito hoy en día y en un futuro venidero, es de vital importancia la innovación en sus servicios junto a la innovación de sus productos, ya que si no van de la mano, el desempeño de uno se verá opacado por el otro y además también cabe mencionar el involucramiento de las nuevas tecnologías que salen día a día, las cuales nos permiten que esta innovación este a la vanguardia.

\subsection{IMPLICACIONES DE LA ORIENTACION PROACTIVA HACIA EL MERCADO, LA COOPERACION Y EL USO DE LAS TIC EN LOS PROCESOS DE INNOVACION DE PRODUCTOS Y SEVICIOS.}

El proceso de innovación para cualquier empresa no es fácil, y más si se habla de innovación de productos, ya que la tasa de fracaso en el momento de un nuevo producto al mercado es alta, sin contar con las significativas afectaciones financieras que implica para la empresa. De ahí que existen tres factores importantes a tener en cuenta en el momento de incursionar al mercado con un producto nuevo, 1) el grado de orientación al mercado, 2) el desarrollo de acciones y cooperación de un buen equipo, y 3) la adecuada implementación de TIC. (Jimenez-Zarco Isabel, 2008).

La orientación proactiva hacia el mercado hace un énfasis en lo importante que es escuchar al cliente final, cuales son realmente las necesidades insatisfechas que tiene y de esta manera poder innovar en nuestros nuevos producto. La cooperación por su parte, hace el análisis de cuán importante es realizar alianzas estratégicas y buenas relaciones con otros stakeholders, quienes nos ayudaran para brindar un mejor producto o servicio a nuestro público. Y evidentemente el uso e implementación de las TIC es algo inherente a nuestro ámbito empresarial, ya que ellas se encuentran en todos los entornos en que un buen negocio innovador se mueve hoy en día.

Finalmente el articulo muestra como se hizo un estudio en empresas españolas del uso de estos tres puntos estratégicos, anteriormente mostrados, y su real efectividad y éxito en empresas mayormente enfocadas a la prestación de servicios, y también, aunque con una pequeña reducción en las empresas innovadoras en productos. 


\subsection{ENFOQUE METODOLOGICO PARA LA INNOVACION EN EMPRESAS}

El concepto de innovación es relativamente un concepto nuevo, ya que en los principios de la industrialización los esfuerzos estaban enfocados en la producción en masa, y posteriormente en procesos especialmente centralizados en la calidad de estos productos. Posteriormente se ha visto un gran incremento en la preocupación de la innovación pero más enfocados hacia el sector secundario por medio de diferentes técnicas que se emplean frecuentemente en los campos de la Administración de empresas tales como, el Brainstorming, Benchmarking, o el Método Delphi entre muchos otros (Chulvi Vicente, 2011).

En este trabajo se propone un sistema nuevo de como atacar y solucionar un problema o una determinada situación que tenga una empresa directamente con el tema de servicio a través de 5 pasos que son el resumen de 4 métodos administrativos que han arrojado buenos resultados, los 5 pasos son: 1. Recopilar información, 2. Procesar información, 3. Detectar Problemas, 4. Buscar Soluciones, y 5. Incluir las soluciones en el marco de innovación.

La principal finalidad que tiene este trabajo es lograr demostrar la importancia que tiene hoy en día las herramientas de innovación en el sector de los servicios en las empresas y como el factor tecnológico cumple un factor fundamental en el ejercicio y desarrollo de estas herramientas en una empresa interesas en ser más competitiva en el sector, con sus competidos y finalmente como puede fidelizar y complacer a sus usuarios finales.

\subsection{EL PROCESO DE COCREACION DE VALOR Y SU IMPACTO EN LA ESTRATEGIA DE INNOVACION EN EMPRESAS DE SERVICIO.}

La preocupación por la innovación en el sector servicios relativamente es reciente, ya que anteriormente, las investigaciones por la innovación se concentraban en el sector industrial o manufacturero, y hasta hace poco se ha empezado a investigar más en el sector terciario, pero lastimosamente estos estudios se han hecho basados en los parámetros de la innovación en el sector industrial, es decir en el producto físico como tal, su diseño, su producción, entre otros. (Ruiz Moreno Antonia, 2014).

La búsqueda de información tanto interna como externa es de suma importancia, ya que son herramientas que brindar un gran apoyo para la retroalimentación de la innovación en especial en el sector servicios. Habría que destacar principalmente tres grandes enfoques o categorías de estudio, como el proceso de innovación de nuevo servicios, el rol de las tecnologías 
de la información y comunicación en los servicios, y los tipos de innovación tanto tecnológicas como organizacionales. (Ruiz Moreno Antonia, 2014)

Hay una especial participación en el sentido de la escucha al consumidor final o el cliente, ya que su participación hace que esta innovación tome un sentido diferenciador entre las empresas de servicios más proactivas. El hecho de estar en constante contacto con este individuo hace que las necesidades que el manifieste puedan verse reflejadas en un mejor servicio para él, lo que se traduce en bienestar para las dos partes. (Ruiz Moreno Antonia, 2014).

Otro factor clave para analizar en este estudio de innovación en el servicio, frente a la innovación en la industria, es que el personal que presta el servicio tiene un papel fundamental en el momento de brindar este servicio, ya que su forma de expresarse y dirigirse al público, su estado de ánimo y los procesos que emplea para prestar el servicio puede variar de un empleado a otro, es por ello que es fundamental estandarizar ciertos procesos o mecanismo que permitan prestar siempre el mejor de los servicios y que no se vea afectado por todos esos factores externos y tan subjetivos.

\section{METODOLOGIA}

En este documento se empleara una metodología de análisis comparativo entre dos órganos prestadores de servicios, con ciertas características similares en el tipo de servicios prestados y en enfocados a nichos de mercados muy similares. Los órganos que se compararan son Barcelona Activa, empresa española con una transcendencia en el mercado por más de 20 años, y la Cámara de Comercio de Bogotá, la cual lleva más de 136 años de fundada.

Esta comparación entre las dos empresas se hará por medio de una lista de chequeo, la cual reflejara algunos puntos comparativos y también los grados de diferencia que tienen las dos empresas para la prestación del servicio al cliente, enfocado más específicamente en el Core business de Barcelona Activa y en uno de los tantos servicios que presta la Cámara de Comercio de Bogotá. 


\begin{tabular}{|c|c|c|c|c|}
\hline \multirow[b]{2}{*}{ PREGUNTA } & \multicolumn{2}{|c|}{ BARCELONA ACTIVA } & \multicolumn{2}{|c|}{ CAMARA DE COMERCIO BTA } \\
\hline & SI & NO & SI & NO \\
\hline 1. Ayuda a la creacion de nuevas empresas & $\mathrm{x}$ & & $\mathrm{x}$ & \\
\hline 2. Experiencia en el mercado local & $\mathrm{x}$ & & $\mathrm{x}$ & \\
\hline 3. Aporta a la generacion de empleo unipersonal & $\mathrm{x}$ & & & $\mathrm{x}$ \\
\hline 4. Hace campanas de promocion? & $\mathrm{x}$ & & $\mathrm{x}$ & \\
\hline 5. Hace seguimiento paso a paso a sus clientes durante el proces & $\mathrm{x}$ & & $\mathrm{x}$ & \\
\hline 6. Tiene espacios de interaccion entre sus clientes? & $\mathrm{x}$ & & & $\mathrm{x}$ \\
\hline 7. Genera redes de ayuda entre sus clientes finales? & $\mathrm{X}$ & & & $\mathrm{X}$ \\
\hline 8. Tiene espacios fisicos de desarrollo o laboratorios? & $\mathrm{x}$ & & & $\mathrm{x}$ \\
\hline 9. Brinda servicio de consejeria de profesional a estudiantes de & $\mathrm{x}$ & & & $\mathrm{X}$ \\
\hline 10. Servicio gratuito & $\mathrm{X}$ & & $\mathrm{x}$ & \\
\hline 11. Buenos resultados en el mecado local e internacional & $\mathrm{x}$ & & $\mathrm{x}$ & \\
\hline 12. Servicio de consejeria no academica a sus usuarios & $\mathrm{x}$ & & & $\mathrm{X}$ \\
\hline
\end{tabular}

\section{RESULTADOS}

Existen varios factores fundamentales que la Barcelona Activa aplica y desarrolla día tras día para lograr su gran objetivo de brindar un excelente e innovador servicio de asesorías especializadas a su segmento objetivo, en el campo de Emprendeduria (emprendimiento), y consecución ágil y eficaz de trabajo. En primer lugar cabe resaltar que sus clientes finales por lo general son personas jóvenes entre los 17 y los 35 años de edad, los cuales tienen algo de los siguientes objetivos en sus cabezas, bien sea desarrollar una idea innovadora como plan de negocio, o conseguir trabajo en un área rentable y que les guste.

Para desarrollar las ideas de innovación la principal herramienta que utiliza Barcelona Activa es el acompañamiento y asesoramiento continuo para con sus clientes finales, ya que por medio de diversas herramientas existe todo el tiempo una asesoría de personal altamente calificado, y de herramientas que les permiten a estos emprendedores poner en marcha sus nuevos planes de negocios. Este seguimiento y acompañamiento en muchas ocasiones está dado por expertos en los diversos campos tales como el financiero, mercadeo, desarrollo de nuevos productos entre muchos otros. (Sans, 2015).

Otro factor sumamente importante y de gran trascendencia que ha intervenido en estos procesos de incubación de empresas desarrollados allí, es el factor tecnológico, ya que por medio de herramientas que Barcelona Activa brinda a sus usuarios, estos nuevos proyectos de Emprendeduria, han sabido disfrutar y aprovechar las herramientas y ayudas tecnológicas que les brindan para el desarrollo de sus propios logros, tal y como se menciona en el artículo (JimenezZarco Isabel, 2008), donde se hace un especial énfasis en la obligada utilización de las TICS en todo procesos de desarrollo de servicio.

Por otra parte en la asesoría que Barcelona Activa hace para la consecución de empleo, existen dos grandes vertientes en las cuales se enfoca, la primera es para personas desempleadas, en edad laboral que buscan un empleo, y acuden a esta empresa buscando una asesoría 
especializada en todo el proceso de acompañamiento de realizar el curriculum, entrevista, tipos de empresas a las cuales aplicar y que tipo de empleo buscar de acuerdo al perfil profesional que tenga cada cliente. Por otra parte realizan un gran servicio con estudiantes de últimos anos escolares para realizar el proceso de guiarlos para que encuentren la mejor opción en cuanto escoger que tipo de carrera universitaria escoger, por medio de acompañamientos guiados por personal altamente calificado, y también por herramientas tecnológicas que permiten un perfilamiento de las aptitudes de cada estudiante para así aconsejarlos en la escogencia de su carrera. (Sans, 2015)

Finalmente Barcelona Activa es una empresa que a través de sus 28 años en el mercado Español, ha logrado que miles de emprendedores de diferentes áreas, hayan logrado sus objetivos y sean clientes finales y usuarios de esta empresa satisfechos con la labor prestada por la empresa en general, que no solo la ven como una empresa prestadora de servicios sino que la ven como un ente que les ayudo a sentar un precedente en sus vidas, y que por tal, tiene un gran reconocimiento tanto a nivel nacional o internacional, por sus múltiples acciones para con la sociedad en general.

\section{CONCLUSIONES}

$\checkmark$ La innovación es un factor que puede generarse tanto de manera disruptiva como de manera incremental, la idea es que haya un factor único en el servicio a innovar, logrando así una trascendencia en la mente del cliente final.

$\checkmark$ Una de las principales claves para prestar un excelente servicio sea en el sector económico que sea, es brindar al cliente final un acompañamiento continuo, para que este en ninguna parte del proceso se sienta solo, y así mismo pueda tener un apalancamiento provechoso.

$\checkmark$ Para poder seguir innovando en el servicio es siempre indispensable escuchar al cliente final, que necesidades no le quedaron satisfechas, que otras necesidades se generaron en el proceso, y que sugerencias o comentarios tiene para con el servicio recibido, ya que de esos comentarios es donde más se enriquece la compañía para seguir mejorando todos los días.

$\checkmark$ La competencia es lo que determina la calidad del servicio que presta una determina compañía, es por eso que es vital que este sea de excelente calidad y también determina la fidelización del cliente a dicha compañía.

$\checkmark$ La innovación es una conservación del conocimiento en valor, es decir, es una consecución constante de valor que se puede dar constantemente en múltiples campos, pero en especial la innovación en el servicio es el motor vital para las futuras empresas, ya que el concepto del cliente final es lo que define la fidelización de este para con la empresa. 
$\checkmark$ Para el caso colombiano es posible tomar como base y como ejes de factores de éxito para empresas prestadoras de servicios privados, el hecho de realizar un constante seguimiento antes, durante y después de prestar el servicio al cliente final, para que de esta manera se logre un buen desempeño y así se generen clientes satisfechos.

$\checkmark$ Es claro que para empresas especializadas en el sector servicios privados estas mejoras son mucho más fácil de incluir y de poner en marcha, que para empresas prestadoras de servicios públicos, más en un contexto tan burocrático y complicado como el colombiano. 


\section{BIBLIOGRAFIA}

Activa, B. (2014). BARCELONA ACTIVA. Obtenido de http://www.barcelonactiva.cat

Ana, M. R. (2010). Memria de Actividades 2010 Barcelona Activa. Barcelona.

Antonio, L. G. (18 de NOVIEMBRE de 2011). http://clio.rediris.es.ISSN:1139-6237. Obtenido de http://clio.rediris.es/n37/oposiciones2/tema20.pdf

Chulvi Vicente, R. L. (2011). Enfoque Metodologico para la innovacion en empresas. DYNA-Ingenieri e Industria, 397-404.

Ena, R. C. (01 de 07 de 2008). Gestiopolis. Obtenido de http://www.gestiopolis.com/metodos-y-tecnicasde-investigacion/

Jimenez-Zarco Isabel, M. P. (2008). Implicaciones de la orientacion hacia el mercado, la coopercion y el uso de las tic en los porcesos de innovacion de productos y servicios. Universia Business Review, 54-67.

K., A. (24 de Febrero de 2014). CreceNegocios. Obtenido de http://www.crecenegocios.com/laimportancia-de-brindar-un-buen-servicio-al-cliente/

Martinez Angel, L. P. (2007). Services Science: a new innovation approach for services providers. Universia Business Review, 120-127.

Nudel, S. K. (16 de Julio de 2009). La impotancia del servicio al cliente. Obtenido de CNNEXPANCION: http://www.cnnexpansion.com/opinion/2009/07/15/la-importancia-del-servicio-al-cliente

Ruiz Moreno Antonia, O. E. (2014). El proceso de cocreacion de valor y su impacto en la estrategia de innovacion en empresas de servicio. Madrid Espana: Intagible Capital.

Sans, M. (2015). Servicio de programas de emprendeduria y empresas, Barcelona Activa. Presentacion de emprendimiento y empresa Barcelona Activa, (pág. 36). Barcelona.

Sundbo, J. (1997). Management of innovation in services. The Service Industries Journal Vol. 17 No. 2. 


\section{ANEXOS}
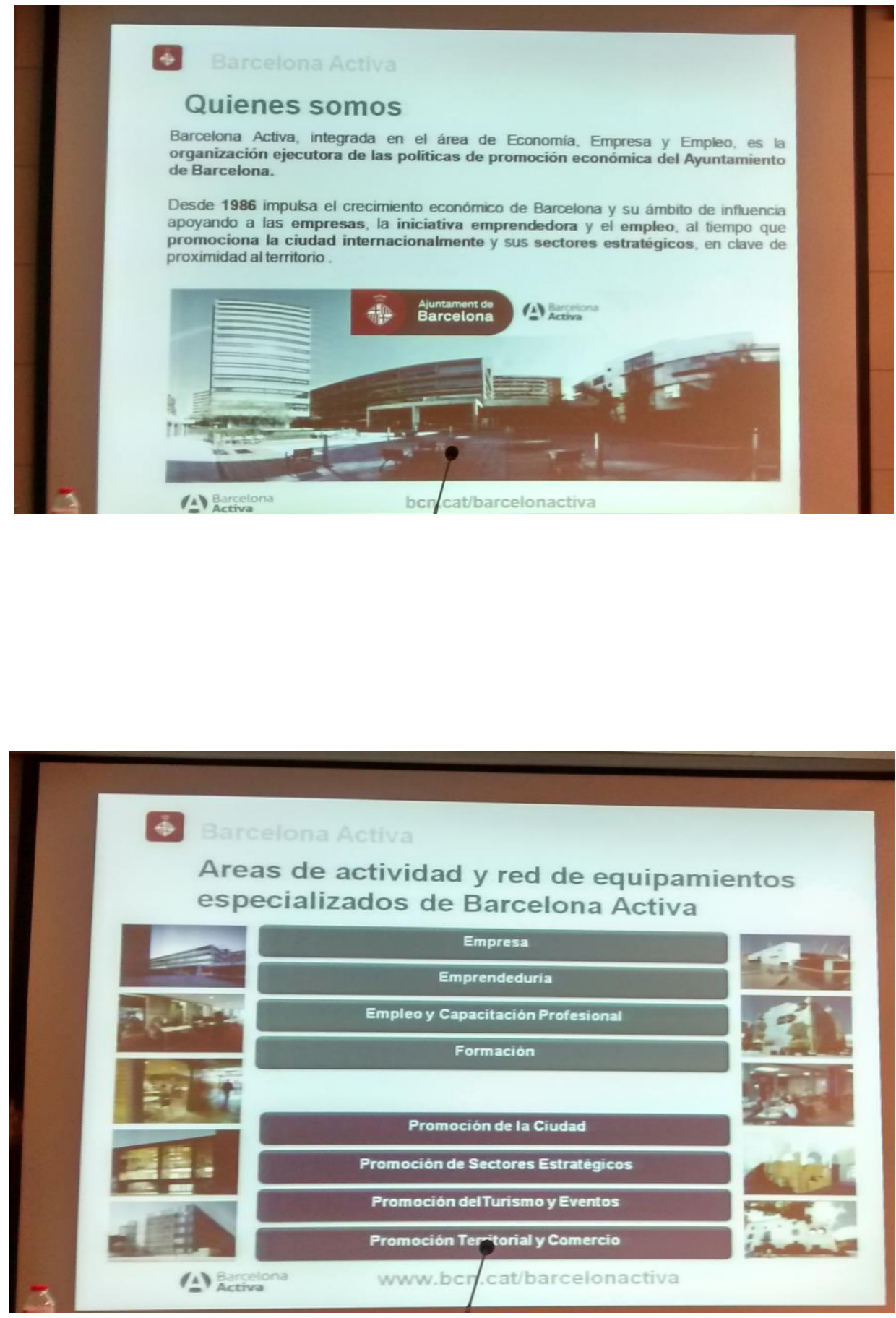

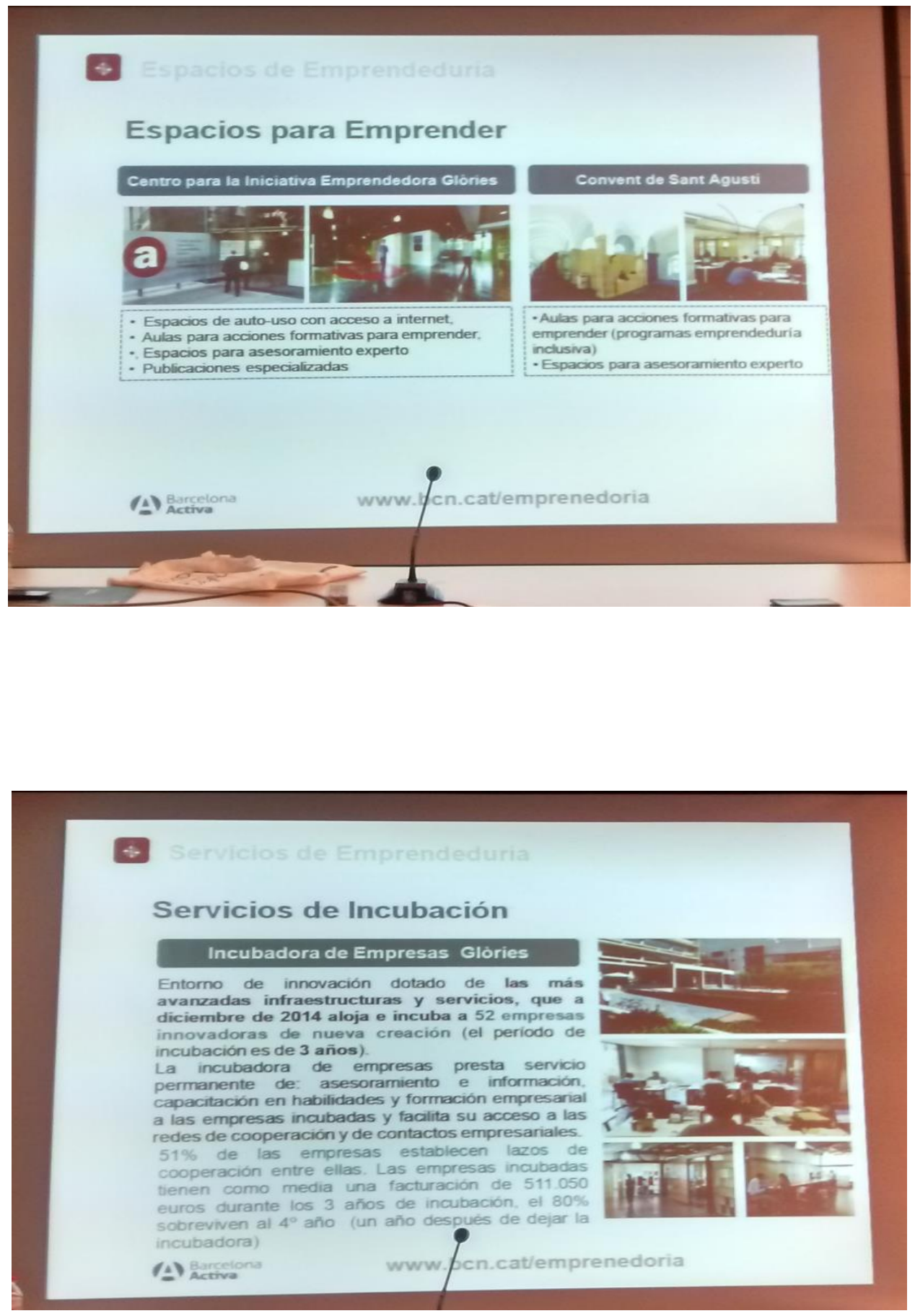

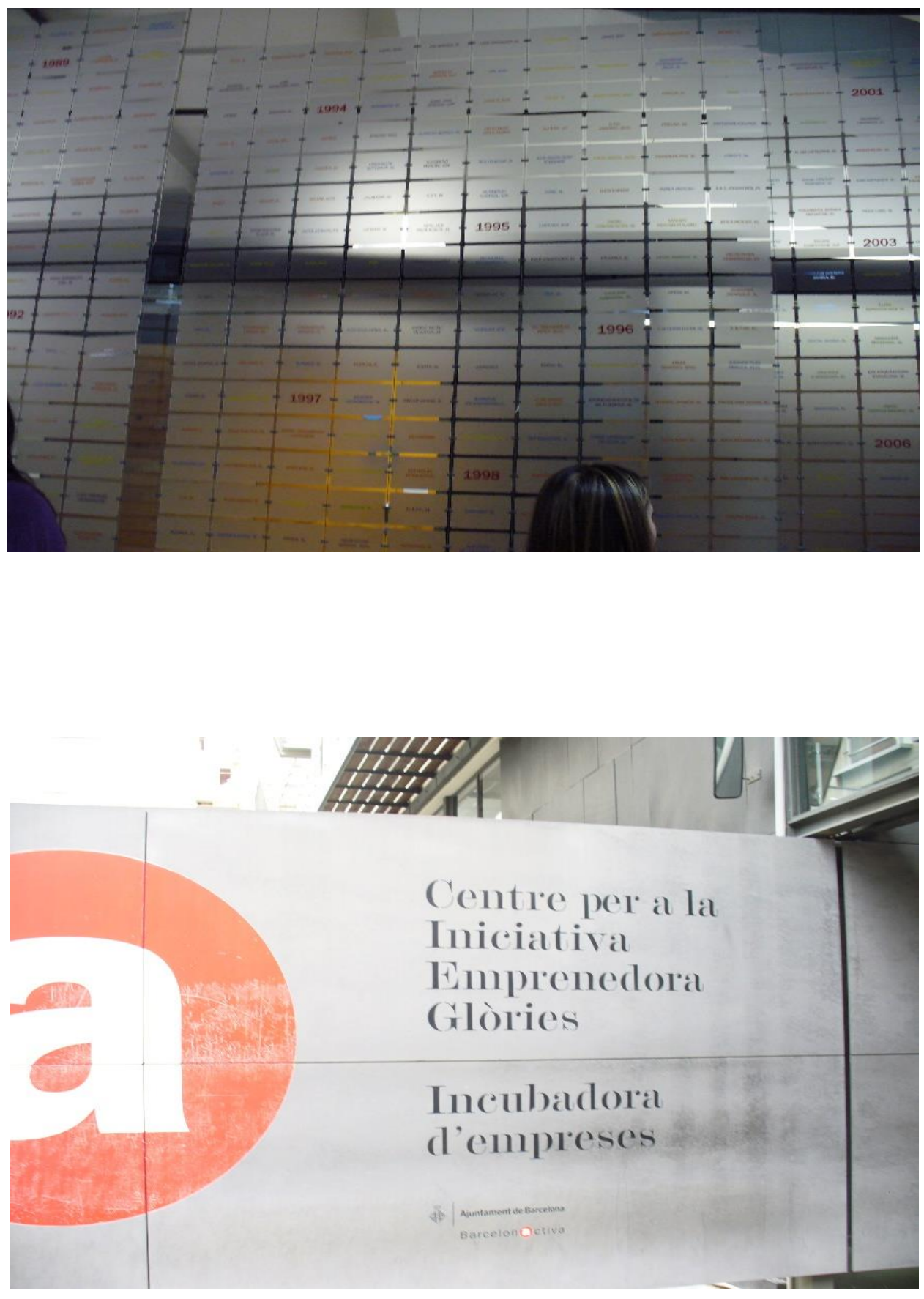
Sesiones formativas para empresas

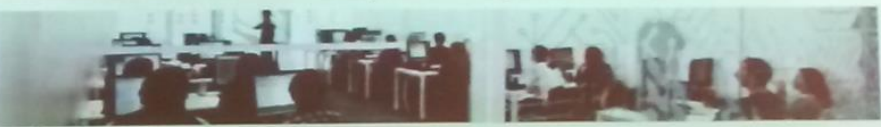

Un abanico de $\mathbf{4 0}$ Cápsulas formativas de corta duración para mejorar las posibilidades de éxito de las empresas en distintas áreas empresariales estratégicas tales como:

- Constitución de la empresa

- Financiación empresanal

Selección de personal

- internacionalización

- Gestión de la innovación

Transmisión de empresas

- Gestón y Estrategia

Marketing y ventas

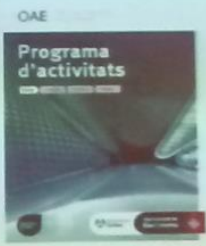

549 sesiones de formación en las que han participado 2.263 empresas en 2014

(A) Activa

www bcn.cat/empresa

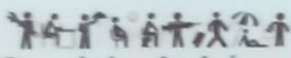

Servicio de búsqueda de empleados

Servicios que proporciona candidatos para las necesidades de personal de empresas de Barcelona y su área metropolitana. Los candidatos se seleccionan entre

- Personas desempleadas formadas por el servicio de empleo de Barcelona Activa que encajan en los requisitos del puesto de trabajo que necesita cubrir la empresa. Durante 2014 se han contratado 500 candidatos.

- Estudiantes de MBA, Post-Grado o grados universitarios en estancia en prácticas (Programa "Pon Talento"). Durante 2014 el programa ha gestionado 110 estancias de estudiantes en empresas.

\section{Empresa-Ocupación}

Plataforma Empresa - Empleo:

Plataforma digital en la que empresas locales pueden encontrar candidatos adecuados para diversos perfiles profesionales, que han cargado en la plataforma digital de empleo de Barcelona Activa uww.bcn.cattreball sus Curriculum Vitaes y la puntuación obtenida en un test de habilidades profesionales de dicha plataforma

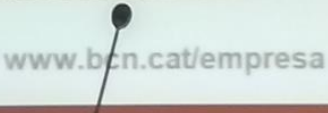

